\title{
SOME IDENTITIES RELATED TO PÓLYA'S PROPERTY $W$ FOR LINEAR DIFFERENTIAL EQUATIONS
}

\author{
ANTON ZETTL
}

1. Introduction. In this note we study relationships between matrix solutions of

$$
X^{\prime}=A X
$$

and

$$
X^{\prime}=B X
$$

where $B=-T^{-1} A^{*} T$ for some constant matrix $T$ satisfying $T^{*} T^{-1}=I$ or $T^{*} T^{-1}=-I$. A familiar example is the case when (1) is the classical vector matrix representation of

$$
L y=y^{k}+\sum_{i=0}^{k-2} a_{i} y^{i}=0 .
$$

Then, for $T=\left((-1)^{i} \delta_{i, k+1-j}\right)$, (2) will represent the adjoint equation

$$
L^{+} y=(-1)^{k} y^{k}+\sum_{i=0}^{k-2}(-1)^{i}\left(\bar{a}_{i} y\right)^{i}=0 .
$$

An application of some of our results to this case will yield that certain sets of fundamental solutions of (3) have property $W$-for a definition see [3] - if and only if certain sets of solutions of (4) have it. We will obtain identities among minors of Wronskians associated with (3) and (4).

2. Determinantal identities. Let $A=\left(a_{i j}\right)$ be a $k \times k$ matrix of continuous complex valued functions on some interval such that $\operatorname{tr} A=0$. Let $T$ be a $k \times k$ constant matrix such that $T^{*} T^{-1}=I$ or $T^{*} T^{-1}=-I$ and let $B=-T^{-1} A^{*} T$. For a given $u$ let $M(t, u)$ and $N(t, u)$ denote the unique matrix solutions of $X^{\prime}=A X$ with $X(u)=I$ and $Y^{\prime}=B Y$ with $Y(u)=I$, respectively.

Lemma 1. $M(t, u) M(u, v)=M(t, v)$.

Proof. This is immediate from the fact that for any nonsingular solution $\phi$ of $X^{\prime}=A X, M(t, u)=\phi(t) \phi^{-1}(u)$, which follows from the

Presented to the Society, November 20, 1964 under the title On harmonic matrices; received by the editors August 1, 1966. 
uniqueness of the solution to the system $X^{\prime}=A X$ with $X(u)=I$. Of course, the same result is valid for $N$.

Lemma 2. $\operatorname{tr} M(t, u)=1=\operatorname{tr} N(t, u)$.

Proof. This follows from the fact that if $X^{\prime}=A X$, then $(\operatorname{det} X)^{\prime}$ $=(\operatorname{tr} A)(\operatorname{det} X)$-see [1, Theorem 7.3, p. 28].

Theorem 1. $M(t, u)=T^{-1} N^{*}(u, t) T$.

Proof. For fixed $u$ let $X(t)=T^{-1 *} M^{*}(t, u) T^{*} N(t, u)$. Then a simple computation yields that $X^{\prime}(t)=0$ and $X(u)=I$. Hence $X(t)=I$. This is equivalent to the theorem in view of $N(u, t) N(t, u)=I$ which follows from Lemma 1.

Let $P(t, u)=\left(P_{i j}(t, u)\right)$ where $P_{i j}(t, u)$ is $(-1)^{i+j}$ times the minor of $N_{i j}(t, u)$ in $N(t, u)$. Then $N(u, t)=N(t, u)^{-1}$ and $\operatorname{det} N(t, u)=1$ imply that $N(u, t)=\bar{P}^{*}(t, u)$. Combining this with Theorem 1 we obtain

Theorem 2. $M(t, u)=T^{-1} \bar{P}(t, u) T$.

This theorem can be made into a much stronger appearing result by using a known fact on adjugate determinants: If $C=\left(c_{i j}\right)$ is a $k \times k$ matrix and $D=\left(d_{i j}\right)$ where $d_{i j}$ is the cofactor of $c_{i j}$, then any algebraic minor of order $r, 1 \leqq r<k$ of $D$ is equal to its algebraic complement in $C$ times $(\operatorname{det} C)^{r-1}$. Combining the above with Theorem 2 we obtain

TheOREM 3. Any $r \times r \quad 1 \leqq r<k$ algebraic minor of $\bar{T} \bar{N}(t, u) \bar{T}^{-1}$ is equal to its algebraic complement in $M(t, u)$.

3. Subwronskians relative to $L$ and $L^{+}$. We now wish to apply some of the above identities to subwronskians of solutions of $L y=0$ and $L^{+} y=0$. This is accomplished by specializing $A$ to

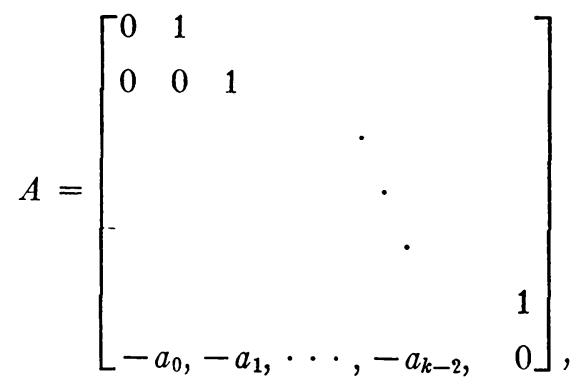

and letting $T=\left((-1)^{i} \delta_{i, k+1-j}\right)$. Now (1) represents (3) and (2) represents (4). Let $y_{i}, z_{j} j=1, \cdots, k$ be solutions of (3) and (4), respectively, such that for some number $a$ 


$$
\begin{aligned}
y_{j}^{i}(a)=1 & \text { if } i=j-1, \\
=0 & \text { if } i \neq j-1,
\end{aligned}
$$

and

$$
\begin{aligned}
z_{j}^{i}(a)=1 & \text { if } i=j-1, \\
=0 & \text { if } i \neq j-1 .
\end{aligned}
$$

An application of Theorem 3 yields:

Corollary 1. Let $\alpha=\left\{\alpha_{1}, \alpha_{2}, \cdots, \alpha_{j}\right\}$ and $\beta=\left\{\beta_{1}, \beta_{2}, \cdots, \beta_{j}\right\}$ be increasing subsequences of $1,2,3, \cdots, k$ and let

$$
\alpha^{\prime}=\left\{k+1-\alpha_{j}, k+1-\alpha_{j-1}, \cdots, k+1-\alpha_{1}\right\}
$$

and

$$
\beta^{\prime}=\left\{k+1-\beta_{j}, k+1-\beta_{j-1}, \cdots, k+1-\beta_{1}\right\} .
$$

Then-using the notation of $[2]-d(M(t, u)[\alpha \mid \beta])=d\left(\bar{N}(t, u)\left(\alpha^{\prime} \mid \beta^{\prime}\right)\right)$.

Among these identities the following ones are of particular interest in connection with Pólya's property $W$. In the notation of [3],

$$
W\left(y_{1}, \cdots, y_{j}\right)=W\left(\bar{z}_{1}, \cdots, \bar{z}_{k-j}\right) .
$$

It is hoped that these identities have applications to other areas, such as, the study of conjugate points, boundary value problems, etc.

\section{REFERENCES}

1. E. A. Coddington and N. Levinson, Theory of ordinary differential equations, McGraw-Hill, New York, 1955.

2. M. Marcus and H. Minc, A survey of matrix theory and matrix inequalities, Allyn and Bacon, Boston, Mass., 1964.

3. G. Polya, On the mean-value theorem corresponding to a given linear differential equation, Trans. Amer. Math. Soc. 24(1922), 312-324.

Louisiana State University 